\title{
Placement and orientation of individual DNA shapes on lithographically patterned surfaces
}

\author{
Ryan J. Kershner ${ }^{1+}$, Luisa D. Bozano', Christine M. Michee ${ }^{1+}$, Albert M. Hung ${ }^{1 \dagger}$, Ann R. Fornof ${ }^{1 '}$, \\ Jennifer N. Cha' ${ }^{1 \dagger}$, Charles T. Rettner', Marco Bersani ${ }^{1 \dagger}$, Jane Frommer', Paul W. K. Rothemund ${ }^{2 \star}$ \\ and Gregory M. Wallraff'^
}

\begin{abstract}
Artificial DNA nanostructures ${ }^{1,2}$ show promise for the organization of functional materials $3^{3,4}$ to create nanoelectronic ${ }^{5}$ or nano-optical devices. DNA origami, in which a long single strand of DNA is folded into a shape using shorter "staple strands ${ }^{16}$, can display 6-nm-resolution patterns of binding sites, in principle allowing complex arrangements of carbon nanotubes, silicon nanowires, or quantum dots. However, DNA origami are synthesized in solution and uncontrolled deposition results in random arrangements; this makes it difficult to measure the properties of attached nanodevices or to integrate them with conventionally fabricated microcircuitry. Here we describe the use of electron-beam lithography and dry oxidative etching to create DNA origami-shaped binding sites on technologically useful materials, such as $\mathrm{SiO}_{2}$ and diamond-like carbon. In buffer with $\sim 100 \mathrm{mM} \mathrm{MgCl}_{2}$, DNA origami bind with high selectivity and good orientation: $\mathbf{7 0 - 9 5 \%}$ of sites have individual origami aligned with an angular dispersion ( \pm 1 s.d.) as low as $\pm 10^{\circ}$ (on diamond-like carbon) or $\pm 2 \mathrm{O}^{\circ}$ (on $\mathrm{SiO}_{2}$ ).
\end{abstract}

The semiconductor industry is currently faced with the challenges of developing lithographic technology for feature sizes below $22 \mathrm{~nm}$ (ref. 7) and exploring new classes of transistors that use carbon nanotubes ${ }^{8}$ or silicon nanowires ${ }^{9}$. A major goal of nanotechnology is therefore to couple the self-assembly of molecular nanostructures with conventional microfabrication. A marriage of these so-called bottom-up and top-down fabrication methods would enable us to register individual molecular nanostructures, to electronically address them, and to integrate them into functional devices. One strategy is to use lithography to make templates onto which discrete components can self-assemble. Examples include the assembly of nanoparticles ${ }^{10,11}$, carbon nanotubes ${ }^{12,13}$ and nanowires ${ }^{14}$. Lithographic templates can also be used to create hierarchical order: the nanostructures they organize can themselves have internal features with dimensions significantly smaller than those of the original template ${ }^{15}$ and can serve as scaffolds for the assembly of still smaller components.

Artificial DNA nanostructures are well suited to this approach. They can be synthesized with attachment groups (such as biotin or single-stranded DNA hooks) at defined locations, which can bind objects such as gold nanoparticles ${ }^{4,16}$. Easily designed in arbitrary shapes, DNA origami typically carry 200 such independently addressable sites at a resolution of $6 \mathrm{~nm}$. Figure 1a depicts the self-assembly of triangular DNA origami in solution (see Supplementary Methods 1) and shows an atomic force micrograph (AFM) of their random deposition on mica, a technique ill-suited for integration with microfabrication. Previous lithographically patterned deposition of organic compounds ${ }^{17}$, single- and doublestranded DNA molecules ${ }^{18-20}$ or DNA nanostructures ${ }^{21}$ has achieved highly selective adsorption, but the molecules were smaller than the lithographic features and thus many molecules packed randomly within a single feature. Dielectrophoretic trapping has captured single origami ${ }^{22}$ between electrodes with a $10 \%$ success rate, but the origami were often folded.

DNA origami are just large enough (here 127-nm-sided triangles are used) that their largest feature, their outline, can match the smallest features generated by lithography. Figure $1 \mathrm{~b}$ outlines how we make use of this property and describes a general method for the placement and orientation of individual DNA origami-single giant molecules-on lithographically patterned surfaces (see Supplementary Methods 2). The idea is to create sticky patches, in the shape and size of a DNA origami, by chemically differentiating lithographic features (see Supplementary Methods 3). Our method begins with a template layer, the layer that will be chemically differentiated on top of a substrate and under a protective layer of photoresist. The photoresist is exposed with the desired pattern of DNA origami binding sites (features), and developed to reveal the template layer at those sites. A dry oxidative etch step is then used to differentiate the template layer and render it sticky for DNA origami. Next, the photoresist is stripped to uncover areas designated non-sticky (background). Finally, nanomolar concentrations of origami are bound to the patterned substrate with high concentrations of divalent cations, here $\mathrm{MgCl}_{2}$ (in Tris-acetate-EDTA [TAE] buffer), with incubation times of minutes to hours. Variables include the choice of template layer (molecular monolayer versus non-monolayer thin film), substrate $\left(\mathrm{SiO}_{2}\right.$ versus silicon), lithographic method (electron-beam versus optical), dry oxidation $\left(\mathrm{O}_{2}\right.$ plasma versus UV-ozone), exposure pattern and the concentration of divalent and monovalent cations.

Figure 2a, b (AFM; see Supplementary Methods 4) shows results using a trimethylsilyl (TMS) monolayer as the template layer, prepared by treating the surface of thermally grown $\mathrm{SiO}_{2}$ with the silylating agent hexamethyldisilazane (HMDS) ${ }^{23}$. Untreated TMS surfaces remain hydrophobic (water contact angle of $\geq 40^{\circ}$ ), whereas those areas exposed to $\mathrm{O}_{2}$ plasma (Fig. 2a) or UV-ozone (Fig. 2b) become hydrophilic (contact angle $<10^{\circ}$, presumably due to silanol groups) and exhibit high selectivity for binding DNA origami. Origami rarely adhere to unoxidized regions of the substrate; for example, none do so in the the $9-\mu \mathrm{m}^{2}$ area from

'IBM Almaden Research Center, San Jose, California 95120, USA, ${ }^{2}$ Department of Bioengineering, Computer Science, and Computation \& Neural Systems, California Institute of Technology, Pasadena, California 91125, USA; Present address: University of Wisconsin, Madison, Wisconsin 53706, USA (R.J.K); The National Academies, Washington DC 20001, USA (C.M.M.); Department of Nanoengineering, University of California, San Diego, California 92093, USA (A.M.H., J.N.C.); Center for Nanoscience, Ludwig-Maximilians Universität, 80799 Munich, Germany (A.R.F.); Dipartimento di Fisica, Università di Padova, I-35131 Padova, Italy (M.B.). *e-mail: pwkr@dna.caltech.edu; gmwall@almaden.ibm.com 


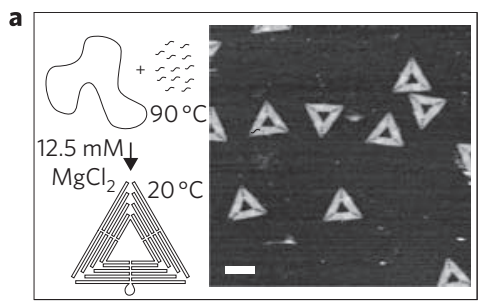

\section{b}

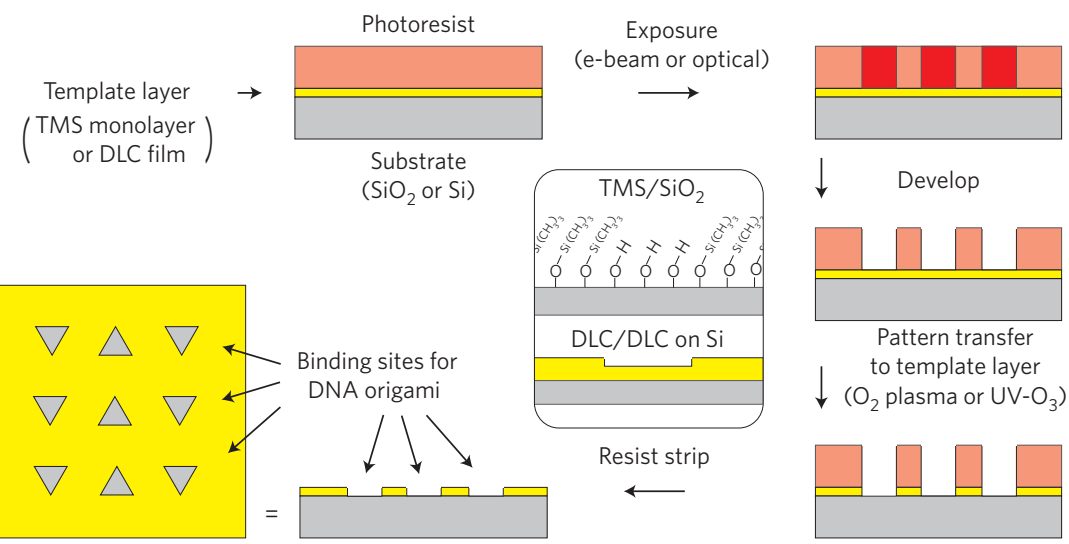

Figure 1 | The problem of random DNA nanostructure deposition and a lithographic scheme for addressing it. a, Synthetic scheme for DNA origami triangles (left) and atomic force microscopy height image (right) showing random deposition on mica. The triangles' edges are $\sim 127 \mathrm{~nm}$; scale bar, $100 \mathrm{~nm}$. b, Fabrication of DNA origami binding sites. The inset highlights differentiation of the background and features (background/features) for the trimethylsilyl (TMS) monolayer and diamond-like carbon (DLC) films. Silanol groups occur in oxidized areas of the TMS monolayers. Features etched into the $\sim 10-n m$ DLC template layer are $0.5-1.5 \mathrm{~nm}$ deep. No silicon is exposed; such surfaces have DLC features on a DLC background, and so we refer to them as 'DLC/DLC'.
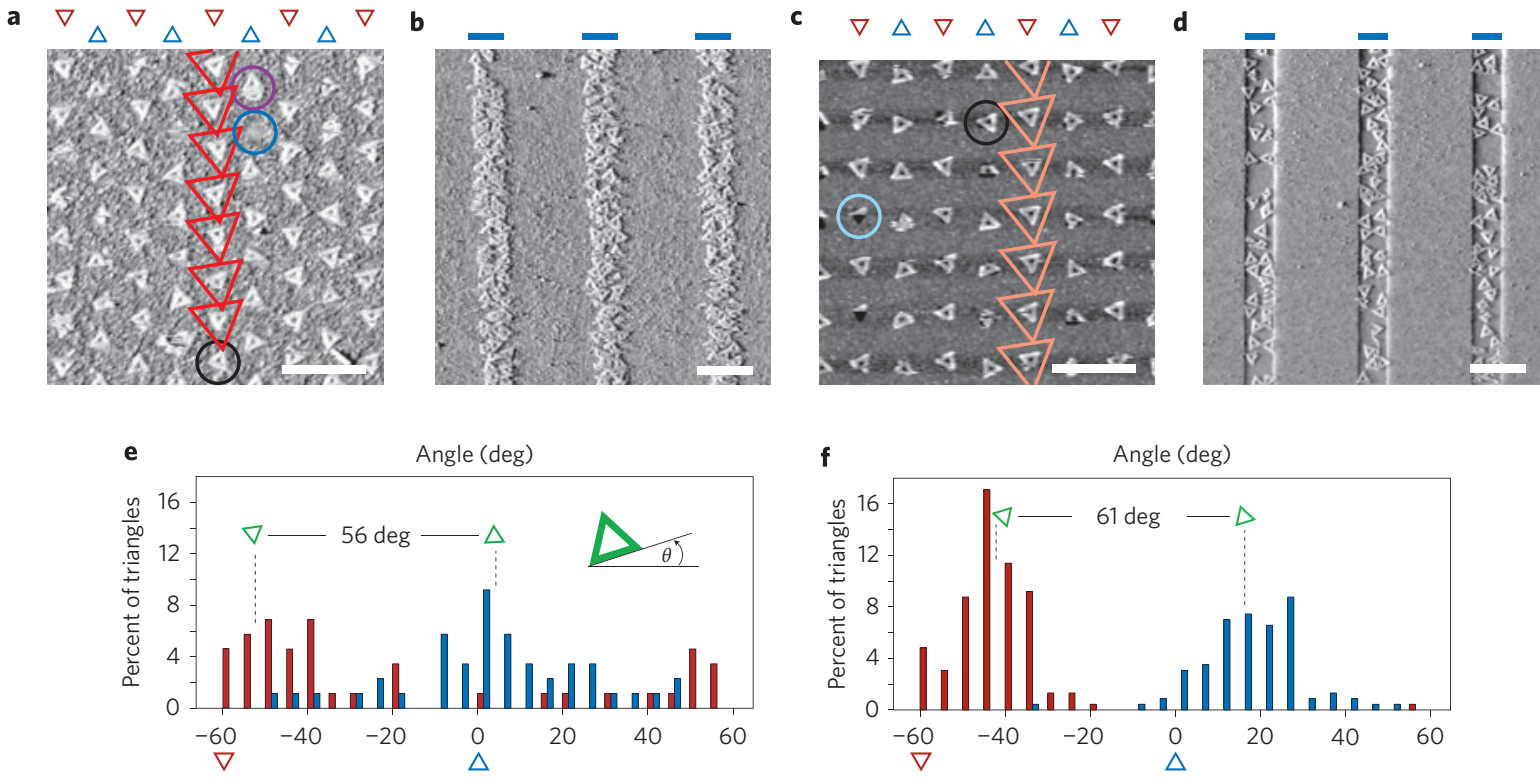

Figure 2 | Alignment of DNA origami on $\mathrm{SiO}_{2}$ and diamond-like carbon (DLC) surfaces. a, Atomic force microscopy (AFM) phase image of DNA triangles on 110 -nm patterned triangles on trimethylsilyl (TMS) $/ \mathrm{SiO}_{2}$. b. AFM phase image of triangles on 300-nm optically patterned lines on $\mathrm{TMS} / \mathrm{SiO}{ }_{2}$ show dense, random placement on lines. c, AFM height image of DNA triangles on $\sim 110 \mathrm{~nm}$ patterned triangles on a DLC/DLC on silicon surface. $\mathbf{d}$, AFM phase image of triangles on $200 \mathrm{~nm}$ electron-beam patterned lines on DLC/DLC; the results are similar to $\mathbf{b}$. Scale bars in $\mathbf{a}-\mathbf{d}, 500 \mathrm{~nm}$. In $\mathbf{b}$ and $\mathbf{d}$ blue bars above the image indicate the dry-oxidized part of the surface, to which triangles stick. In a and $\mathbf{c}$, blue and red triangles above the image indicate the orientation of oxidized triangular patches on the surface in each column below. Red/pink triangles superimposed on the image: DNA triangles with roughly the correct orientation. Black circles: triangles of incorrect orientation. Purple circle: a site at which two triangles have bound. Blue circles: empty site (a) or site at which an origami is being removed by the AFM tip (c). e,f, Measured orientations from larger 3 and $5 \mu \mathrm{m}$ areas of $\mathbf{a}$ and $\mathbf{c}$. Red and blue triangles mark desired down and up orientations. Red bars indicate the fraction of triangles at a particular angle, measured in down-oriented columns; blue bars indicate the same for up-oriented columns. $-60^{\circ}$ and $60^{\circ}$ are equivalent. Green triangles indicate peaks in the measured orientation.

which Fig. 2a was taken (see Supplementary Fig. S1) or the $9-\mu \mathrm{m}^{2}$ area of Fig. 2b. Fig. 2b shows origami bound to 300-nm optically patterned lines; multiple origami bind at random positions and with random orientation. Figure 2 a shows origami triangles on $\sim 110$-nm electron-beam patterned triangular binding sites. The occupancy and alignment were measured at 136 sites (see Supplementary Methods 5, Figs. S1, S2, S20a). In total, $88 \%$ of sites bound a single origami and $7 \%$ of sites bound pairs ('doublebinding events'). In columns of sites designed to have an 'up' orientation of $0^{\circ}$, single origami had an orientation \pm angular dispersion of $\theta_{\triangle}=3.6 \pm 22^{\circ}$ (mean \pm 1 s.d.; $N_{\triangle}=43$; Fig. $2 \mathrm{e}$, blue bars); in columns of 'down' sites at $-60^{\circ}$, the orientation was $\theta_{\nabla}=-52 \pm 20^{\circ}\left(N_{\nabla}=44\right.$; Fig. 2 e, red bars). Similar results $(70 \%$ single origami/site, $\theta_{\triangle}=18 \pm 21^{\circ}, \theta_{\nabla}=-49 \pm 22^{\circ} ; N_{\triangle}=40$, $N_{\nabla}=40$ ) were obtained in an independent experiment, using a different $\mathrm{O}_{2}$ plasma etch tool (see Supplementary Figs S3, S4, S20b) although the fraction of double-binding events (30\%) was much higher. 
To understand the significance of experimental angular distributions we (i) estimated the expected standard deviation (s.d.) for randomly oriented distributions of size $N$ by averaging computer-generated distributions, (ii) estimated the standard deviation of these $\overline{\text { s.d. }}$ (s.d."), and (iii) used the $\overline{\text { s.d. }}$ and s.d." to calculate the probability ( $P$-value) that a sample of random orientations would have equal or lower angular dispersion $( \pm 1$ s.d.) than a given experimental distribution. As $N$ ranged from 15 to 240 the $\overline{\text { s.d. }}$ for $10^{5}$ computer-generated distributions ranged from 30 to $33^{\circ}$, with s.d." from 2.8 to $0.66^{\circ}$. Experimental distributions for all samples of origami on triangular features with $100 \mathrm{mM} \mathrm{MgCl}_{2}$ (including those with DLC/DLC below) had angular dispersions $\leq 22^{\circ}$, several s.d." away from the s.d. for matching $N$. Thus $P$-values were all small $\left(\leq 5 \times 10^{-10}\right)$, and these distributions were deemed significantly different from randomly oriented samples. Origami on 350-nm line features (see Supplementary Figs S5, S6, S21a) had an angular dispersion of $33.3^{\circ}$, consistent with randomly oriented samples $(P=0.45$ when compared with $\overline{\text { s.d. }}$ of $33.2 \pm 0.66$ for $N=240$ ).

The creation of TMS monolayers with HMDS is simple and widely performed in the semiconductor industry, but TMS monolayers are fragile and hydrolyse quickly. Non-monolayer thin films can be more durable, and allow the addition of topography to the template; that is, binding sites can have a well-defined depth. We chose diamond-like carbon (DLC) ${ }^{24}$ as a prototype non-monolayer thin film because it is chemically resistant, hard, smooth, hydrophobic and easily etched by $\mathrm{O}_{2}$ plasma. DLC films $(10 \mathrm{~nm})$ on silicon were fabricated ${ }^{25}$, electron-beam patterned with either $\sim 110 \mathrm{~nm}$ triangles (Fig. 2c) or 200-500 nm lines (for example, Fig. 2d), and $\mathrm{O}_{2}$ plasma-etched to a depth of $0.5-1.5 \mathrm{~nm}$. Origami stuck with high selectivity to binding sites. It was unnecessary to etch down and oxidize the silicon substrate: the dry-etched DLC was itself attractive for origami. For 304 triangular features on sites of $\sim 1 \mathrm{~nm}$ depth (see Supplementary Figs. S7, S8), measurement of site occupancy gave results (95\% single origami) similar to those on $\mathrm{TMS} / \mathrm{SiO}_{2}$ except that the fraction of double-binding events $(0 \%)$ was much lower, perhaps because binding sites are topographically as well as chemically differentiated. No alignment was observed on $200-\mathrm{nm}(N=68)$ or $500-\mathrm{nm}(N=203)$ line features (best-fit peaks gave $\pm 31^{\circ}$ and $\pm 32^{\circ}$, consistent with samples of random angles; see Supplementary Figs S16-S19, S21b,c), but alignment on triangular features $\left(\theta_{\triangle}=18 \pm 12^{\circ}, \theta_{\nabla}=-43 \pm 8^{\circ}, N_{\triangle}=96, N_{\nabla}=132\right.$; Fig. 2f $)$ had half the dispersion measured on $\mathrm{TMS} / \mathrm{SiO}_{2}$. Although these triangles are consistently oriented, and show a $61^{\circ}$ difference between peak orientations, they have an unexpected offset $\left(\sim 17^{\circ}\right)$ with respect to the desired orientation. Similar offsets appear in other data and may be an artifact of imaging under buffer, which allows the triangles to move. We have not explored the effect as a function of scan angle, substrate tilt or other parameters. DLC/DLC substrates could be reused after piranha cleaning $\left(3: 1 \mathrm{H}_{2} \mathrm{SO}_{4}: 30 \% \mathrm{H}_{2} \mathrm{O}_{2}\right)$ albeit with somewhat degraded alignment $\left(\theta_{\triangle}=19 \pm 18^{\circ}, \theta_{\nabla}=-42 \pm 13^{\circ}, N_{\triangle}=94\right.$, $N_{\nabla}=71$; see Supplementary Figs. S9, S10, S20d). Similar results $\left(\theta_{\triangle}=18 \pm 13^{\circ}, \theta_{\nabla}=-44 \pm 13^{\circ} ; N_{\triangle}=148, N_{\nabla}=112\right)$ were obtained on slighty deeper $(\sim 1.4 \mathrm{~nm})$ features (see Supplementary Figs S11, S12 and S20e).

More generally, there are many potential effects of binding site size on site occupancy and alignment, and we have yet to measure them quantitatively. However, a $\sim 2 \mathrm{~h}$ movie (Fig. 3; see also Supplementary Movie, Figs S22 and S23) of triangles binding to sites with a variety of sizes from 90 to $400 \mathrm{~nm}$ suggests that (i) single origami bind with qualitatively good orientation over a range of sizes, but (ii) the more undersized the site, the worse the alignment, and (iii) the more oversized the site, the greater the probability of multiple origami binding. The movie also shows the dynamic nature of binding under fluid-mode AFM imaging. Although the percentage of filled sites reached a steady state within
Edge length of triangular feature (nm)

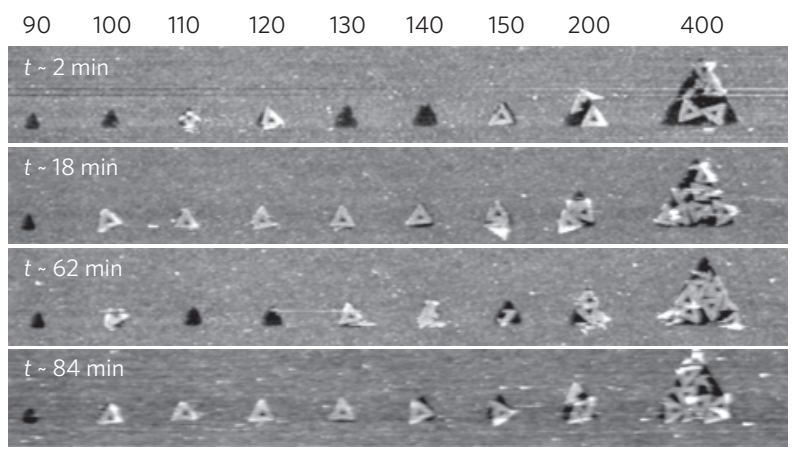

Figure 3 | Dynamic binding of DNA origami under atomic force microscopy (AFM). Frames from an AFM height movie show the dynamics of binding at sites of different sizes. AFM is performed under 10× TAE buffer with $100-125 \mathrm{mM} \mathrm{MgCl}_{2}$.

several minutes, and remained approximately constant for a couple hours, the specific location of the unfilled sites varied constantly. In series of frames showing the dissociation of an intact origami, later frames often show partially-imaged triangles (for example, a single tip or edge). Thus partially-imaged origami in single-image data (data not from movies) may be evidence of dissociation, rather than origami fragmentation. How much the observed rates of association and dissociation reflect reversible binding, and how much they reflect tip-sample interaction is unknown; however, reversibility must be high enough to allow the observed quality of alignment.

The binding of two or more origami at oversized sites suggests the question of whether integral numbers of origami might closepack on sites of integral triangle unit area $\left(A_{\triangle} \approx 7,000 \mathrm{~nm}^{2}\right.$ for 127-nm DNA triangles) with different shapes. This is explored in Fig. 4 with a collection of polygonal sites designed to bind two origami per parallelogram, three per trapezoid, and so on. Binding sites are slightly undersized $\left(121 \mathrm{~nm}, 6,300 \mathrm{~nm}^{2}\right)$ and missing triangles or partial triangles are the most common defects. Nevertheless, the number of origami per unit area is linear whether whole triangles $\left(0.76\right.$ origami $\left./ A_{\triangle}, R^{2}=0.98\right)$ or partial triangles $\left(0.99\right.$ origami $\left./ A_{\triangle}, R^{2}=0.99\right)$ are counted, or whether slightly undersized or oversized features are used (see Supplementary Figs S24-S28). Close-packing is occasionally observed (yellow outlines, Fig. 4b), but the frequency of random arrangements suggests that the use of single origami binding sites with small spacings between them may be the best way to create small, complex patterns of origami.

The adsorption of origami to dry etch-oxidized features is highly sensitive to salt concentration on both $\mathrm{TMS} / \mathrm{SiO}_{2}$ and DLC/DLC surfaces. No binding was observed when origami were deposited in $12.5 \mathrm{mM} \mathrm{MgCl}_{2}$ with $1 \times \mathrm{TAE}$ buffer $(40 \mathrm{mM}$ Tris acetate, $1 \mathrm{mM}$ EDTA, $2 \mathrm{mM} \mathrm{Na}^{+}$), the conditions under which DNA origami are formed and bind well to mica. A $~ 10$-fold more concentrated buffer (100-125 mM MgCl$/ 2 / 9-10 \times$ TAE) gave quantitative adsorption of origami to binding sites on both surfaces. On DLC/DLC, a further $\sim 10$-fold increase in $\mathrm{MgCl}_{2}$ ( $1 \mathrm{M}$, leaving monovalent cations constant at $9-10 \times$ TAE; see Supplementary Figs S14, S13) resulted in a stark reduction in single origami adsorption (9\% of 328 sites, down from 95\% in Supplementary Fig. S8), gave a high rate of double-binding (1.5\% of total sites but $9 \%$ of bound sites, up from $0 \%$ ) and removed orientation selectivity (see Supplementary Fig. S21d). On TMS $/ \mathrm{SiO}_{2}$, binding on line features also decreased as $\mathrm{MgCl}_{2}$ increased to $1 \mathrm{M}$ (see Supplementary Fig. S27). These decreases in adsorption are similar to that observed for duplex DNA on mica when $\mathrm{Mg}^{2+}$ is increased to $1 \mathrm{M}$ (ref. 26). 


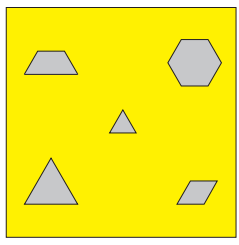

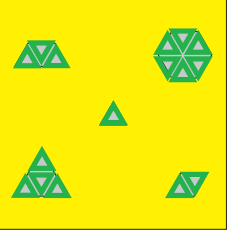

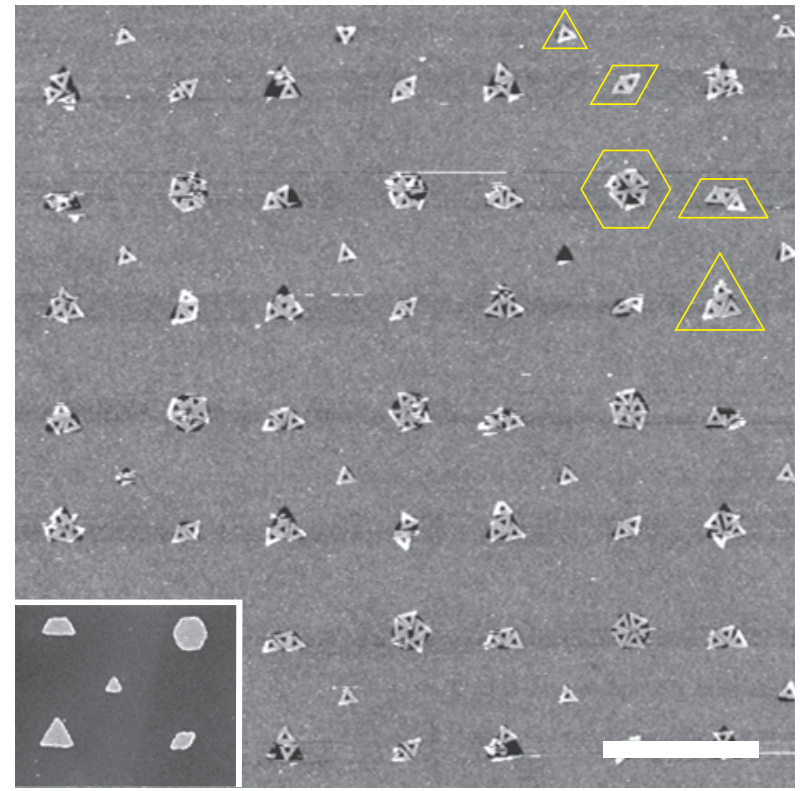

Figure 4 | Placement of triangles onto a variety of shapes.

a, Scheme showing how one, two, three, four or six DNA triangles are expected to bind sites with the shape of a unit triangle, rhombus, trapezoid, double-sized triangle and hexagon. $\mathbf{b}$, AFM of results on DLC/DLC; unit triangle is $\sim 120 \mathrm{~nm}$. Yellow outlines highlight examples with well-oriented triangles. Inset: Scanning electron micrograph of $\mathrm{Au} / \mathrm{Cr}$ islands on $\mathrm{Si}$ created by liftoff using a similar electron-beam pattern. These liftoffgenerated patterns are used to calibrate the electron-beam dose (i) to optimize the sharpness of shapes and (ii) to ensure complete resist removal (if incomplete, islands do not adhere). Scale bar, $1 \mu \mathrm{m}$

The point of zero charge of $\mathrm{SiO}_{2}$ is $\sim \mathrm{pH} 2$ (refs 27,28 ), so features are expected to bear a negative charge if under our experimental buffer $\left(\mathrm{pH}\right.$ 8.3) in the absence of $\mathrm{Mg}^{2+}$ ions. Thus an adsorption mechanism consistent with our data is charge inversion (or overcharging) in the electrical double layer ${ }^{29,30}$ of $\mathrm{SiO}_{2}$ features at $100 \mathrm{mM} \mathrm{MgCl}$ and charge inversion of DNA at $1 \mathrm{M} \mathrm{MgCl}_{2}$ (ref. 26). Charge inversion can occur when a negatively charged surface (of the features or DNA) binds a sufficient number of divalent magnesium ions to become positively charged. At an intermediate $\mathrm{Mg}^{2+}$ concentration at which charge inversion occurs on the features, negatively charged DNA structures are bound. At a high $\mathrm{Mg}^{2+}$ concentration, the DNA structures undergo charge inversion, and are repelled from the positive (charge-inverted) features. Charge inversion on silica has been observed under $350 \mathrm{mM} \mathrm{Mg}^{2+}$ at pH 7.5 using streaming current measurements ${ }^{31}$. Our buffer is at $\mathrm{pH} 8.3$, and the adsorption of metal ions on silanol-terminated silicon generally increases linearly with $\mathrm{pH}$ (ref. 32), so $\mathrm{SiO}_{2}$ charge inversion at the somewhat lower $\mathrm{Mg}^{2+}$ concentration $(\sim 100 \mathrm{mM})$ used here seems reasonable. In addition to charge inversion, explicit salt bridges between silanols, $\mathrm{Mg}^{2+}$ ions and phosphates on the DNA backbone may contribute to origami binding; the highdensity, two-dimensional arrangment of adjacent phosphate groups on the face of origami might facilitate this binding mechanism.

Oxidation of diamond films results in hydroxyl, carbonyl or ether groups ${ }^{33}$ creating Lewis basic or anionic sites that may bind magnesium ions and cause analogous charge inversion and salt bridges in the case of DLC features. However, both features and background in the DLC/DLC system show similar evidence of oxidation by X-ray photoelectron spectroscopy (see Supplementary Methods 3), so which chemical species mediate the difference between origami-binding features and the background have yet to be determined. Despite ignorance of the exact binding mechanism, the success of $\mathrm{O}_{2}$ plasma etching in creating binding sites on two disparate surfaces suggests that our method may be generalized to other template layers and substrates.

Recent extension of our method to organize gold nanoparticles ${ }^{34}$ lies along the path towards organizing nanodevices for nanoelectronics. What else might one do with 'bottom-up keys' that fit 'topdown locks'? This technology may find use wherever there is a need to place individual molecules (or a defined number) in a pattern on a surface-one merely needs to couple those molecules to DNA origami. Thus, single-molecule biophysics may benefit. Individual copies of a fluorescently labelled protein could be spaced on a grid for which micrometre-scale dimensions would clearly separate their images in an optical microscope. This would simplify data collection because the experimenter would know where the proteins are located. The triangular origami used here can stick to a triangular binding site in six different ways (three rotations, face up and face down), and so any molecules they might carry would occur in the same range of orientations. This is not a fundamental limit to our approach; when used to position and orient suitably asymmetric DNA origami our method may achieve absolute orientational control over molecules using topdown lithography.

Received 9 March 2009; accepted 7 July 2009;

published online 16 August 2009

\section{References}

1. Seeman, N. C. Nucleic-acid junctions and lattices. J. Theoret. Biol. 99, 237-247 (1982).

2. LaBean, T. H. \& Li, H. Constructing novel materials with DNA. Nanotoday 2, 26-34 (2007).

3. Braun, E., Eichen, Y., Sivan, U. \& Ben-Yoseph, G. DNA-templated assembly and electrode attachment of a conducting silver wire. Nature 391, 775-778 (1998).

4. Le, J. D. et al. DNA-templated self-assembly of metallic nanocomponent arrays on a surface. Nano Lett. 4, 2343-2347 (2004).

5. Kennen, K., Berman, R. S., Buchstan, E., Sivan, U. \& Braun, E. DNA-templated carbon nanotube field effect transistor. Science 302, 1380-1382 (2003).

6. Rothemund, P. W. K. Folding DNA to create nanoscale shapes and patterns. Nature 440, 297-302 (2006)

7. International Technology Roadmap for Semiconductors, 2007 edition (http:// www.itrs.net/Links/2007ITRS/Home2007.htm).

8. Zhang, L. et al. Assessment of chemically separated carbon nanotubes for nanoelectronics. J. Am. Chem. Soc. 130, 2686-2691 (2008).

9. Lu, W. \& Lieber, C. M. Nanoelectronics from the bottom up. Nat. Mater. 6, 841-850 (2007)

10. Cui, Y. et al. Integration of colloidal nanocrystals into lithographically patterned devices. Nano Lett. 4, 1093-1098 (2004).

11. Malaquin, L., Kraus, T., Schmid, H., Delamarche, E. \& Wolf, H. Controlled particle placement through convective and capillary assembly. Langmuir 23, 11513-11521 (2007).

12. Wang, Y. et al. Controlling the shape, orientation, and linkage of carbon nanotube features with nano affinity templates. Proc. Natl Acad. Sci. USA, 103, 2026-2031 (2006).

13. Seemann, L., Stemmer, A. \& Naujoks, N. Local surface charges direct the deposition of carbon nanotubes and fullerenes into nanoscale patterns. Nano Lett. 7, 3007-3012 (2007).

14. Fan, Z. et al. Wafer-scale assembly of highly ordered semiconductor nanowire arrays by contact printing. Nano Lett. 8, 20-25 (2008).

15. Cheng, J. Y., Ross, C. A., Smith, H. I. \& Thomas, E. L. Templated self-assembly of block copolymers: top-down helps bottom-up. Adv. Mater. 18, 2505-2521 (2006).

16. Zheng, J. et al. Two-dimensional nanoparticle arrays show the organizational power of robust DNA motifs. Nano Lett. 6, 1502-1504 (2006).

17. Losilla, N. S. et al. Sub-50 nm positioning of organic compounds onto silicon oxide patterns fabricated by local oxidation nanolithography. Nanotechnology 19, 455308 (2008) 
18. Chi, P.-Y., Lin, H.-Y., Liu, C.-H. \& Chen, C.-D. Generation of nano-scaled DNA patterns through electro-beam induced charge trapping. Nanotechnology 17, 4854-4858 (2006).

19. Tanaka, S., Taniguchi, M. \& Kawai, T. Selective adsorption of DNA onto $\mathrm{SiO}_{2}$ surface in $\mathrm{SiO}_{2} / \mathrm{SiH}$ pattern. Jpn. J. Appl. Phys. 43, 7346-7349 (2004).

20. Djenizian, T., Balaur, E. \& Schmuki, P. Direct immobilization of DNA on diamond-like carbon nanodots. Nanotechnology 17, 2004-2007 (2006).

21. Sarveswaran, K., Hu, W., Huber, P. W., Bernstein, G. H. \& Lieberman, M. Deposition of DNA rafts on cationic SAMs on silicon [100]. Langmuir 22, 11279-11283 (2006).

22. Kuzyk, A., Yurke, B., Toppari, J. J., Linko, V. \& Törmä, P. Dielectrophoretic trapping of DNA origami. Small 4, 447-450 (2008).

23. Moreau, W. M. Semiconductor Lithography Principles, Practices and Materials (Plenum Press, 1998).

24. Ferrari, A. C. Diamond-like carbon for magnetic storage disks. Surf. Coat. Technol. 180, 190-206 (2004).

25. Druz, B. et al. Ion beam deposition of diamond-like carbon from an RF inductively coupled $\mathrm{CH}_{4}$-plasma source. Surf. Coat. Technol. 86-87, 708-714 (1996).

26. Pastŕe, D. et al. Anionic polyelectrolyte adsorption on mica mediated by multivalent cations: a solution to DNA imaging by atomic force microscopy under high ionic strength. Langmuir 22, 6651-6660 (2006).

27. Zhang, X. G. Electrochemistry of Silicon and its Oxide (Springer, 2001)

28. Ermakova, L. E., Sidorova, M. P. \& Bogdanova, N. F. Influence of the structure of boundary layers and the nature of counterions on the position of the isoelectric point of silica surfaces. Colloid J. 68, 411-416 (2006).

29. Grosberg, A. Y., Nguyen, T. T. \& Shklovskii, B. I. Colloquium: the physics of charge inversion in chemical and biological systems. Rev. Mod. Phys. 74, 329-345 (2002)
30. Lyklema, J. Overcharging, charge reversal: chemistry or physics? Colloids Surf. A 291, 3-12 (2006)

31. van der Heyden, F. H. J., Stein, D., Besteman, K., Lemay, S. G. \& Dekker, C. Charge inversion at high ionic strength studied by streaming currents. Phys. Rev. Lett. 96, 224502 (2006).

32. Loewenstein, L. M. \& Mertens, P. W. Adsorption of metal ions onto hydrophilic silicon surfaces from aqueous solution: effect of pH. J. Electrochem. Soc. 145, 2841-2847 (1998)

33. Szunerits, S. \& Boukherroub, R. Different strategies for functionalization of diamond surfaces. J. Solid State Electrochem. 12, 1205-1218 (2008).

34. Hung, A. M. et al. Spatially-directed assembly of gold nanoparticles on lithographically patterned DNA origami. Nature Nanotech. (Submitted).

\section{Acknowledgements}

This work was supported by National Science Foundation grants CCF/NANO/EMT0622254 and -0829951 and the Focus Center Research Program (FCRP). Center on Functional Engineered Nano Architectonics (FENA) Theme 2. P.W.K.R thanks Microsoft Corporation for support. The authors thank D. Miller for performing XPS measurements, B. Davis for optical lithography, D. Hoffman for sample preparation, and M. Sanchez, M. Hart and F. Houle for helpful discussions.

\section{Author contributions}

All authors contributed significantly to the work presented in this paper.

\section{Additional information}

Supplementary information accompanies this paper at www.nature.com/ naturenanotechnology. Reprints and permission information is available online at http://npg nature.com/reprintsandpermissions/. Correspondence and requests for materials should be addressed to P.W.R. and G.MW. 